\title{
Electrooptical properties of liquid crystal n-pentil-n'-cyanobifenil with J-aggregates of astrofloxine
}

\author{
Yu. P. Piryatinski, M. S. Furier, V. G. Nazarenko \\ Institute of Physics of the National Academy of Sciences of Ukraine, 46 prosp. Nauki, 03039 Kiev, Ukarine
}

\begin{abstract}
It is shown that organic nanocrystallites - J-aggregates - can be formed and oriented in a liquid crystalline matrix. External electric field can reorient and destroy these nanocrystallites. Transmission and photoluminescence spectra of astrofloxine solutions in the nematic liquid crystal are investigated in d.c. and a.c. electric fields with the frequencies of $1 \mathrm{~Hz}-1 \mathrm{kHz}$ and amplitudes $F=1.2 \times 10^{4} \mathrm{~V} \cdot \mathrm{cm}^{-1}$. Intense photoluminescence was observed at two-photon excitation. Efficiency of IR laser radiation transformation into visible photoluminescence was as high as $10 \%$.
\end{abstract}

Keywords: liquid crystal, nanocrystallite, photoluminescence, two-photon excitation.

Paper received 16.11.00; revised manuscript received 30.01.01; accepted for publication 12.12.01.

\section{Introduction}

Nematic liquid crystals (LC) are often used as matrices for parallel orientation of elongated dye molecules [1]. In an external electric field these LC exhibit pronounced electrooptical properties, i.e. the changes of absorption spectra (electrochromic effect) and photoluminescence (PL) spectra. Electrchromic effect is caused by two different physical processes [2]:

i) external electric field changes the orientation of molecules and, hence, the absorption spectrum intensity,

ii) the field changes the distances between energy levels of the molecules, shifts the absorption bands and varies the transition moments.

Changes in the absorption spectra observed in optical experiments are not related to the shift due to Stark effect and separate lines splitting. They are caused by the extinction coefficient variation $[2,3]$. The spectral features of a dye are essentially influenced by the medium where it is dissolved or dispersed. The medium also determines the phase state of the dye and evolution of its spectrum in the electric field. It was shown recently [4] that organic nanocrystallites - J-aggregates - can be formed and oriented in a LC matrix.
At present the above-mentioned organic and inorganic semiconductor particles of sub-micron size are widely investigated. E.g., in [5] it was shown that J-aggregates of pseudoisocyanines have very large nonlinear cubic susceptibility [5]. Elongated (rod-like) shape and large electric dipole along the aggregate axis are the key properties for the creation of «macroanisotropic» media on the base of aligned J-aggregates. One of the possible methods to create «microanisotropy» is to align the aggregates in the flow of solutions [6] and polymers. But this «microanisotropy» is static and uncontrollable. The present paper deals with the investigation of J-aggregates behavior in a nematic LC that has a long-range order, on the one hand, and can be reoriented in the electric field, on the other hand.

In the present study we continue the investigations of electrooptical properties of the system of J-aggregates of the dye in the LC that have been started in [4]. The orienting medium for J-aggregates is the LC n-pentil-n'cyanobifenil (5CB). To obtain J-aggregates we used the dye of the pseudoisocyanine family - astrofloxine (AF). Nanocrystals of AF molecules in LC matrix have the spectra and PL lifetime that differs from those of the AF molecules. External electric field can reorient and destroy J-aggregates. The influence of the electric fields of various frequencies and amplitudes is investigated. The role of the surface in 
these phenomena is analyzed. High efficiency of two phonon transformation of IR laser radiation into visible light was found for J-aggregates of very long AF in the 5CB LC matrix.

\section{Experimental}

For the preparation of AF solutions in LC matrix we used npentil-n'-cyanobifenil produced by the firm Merk (Germany). $5 \mathrm{CB}$ has the temperatures of the phase transitions crystalLC and LC-isotropic fluid equal to 22.5 and $36.5 \mathrm{C}$, respectively. To obtain necessary solutions, AF has been at first dissolved in toluene, then mixed with LC and then heated to $70-80 \mathrm{C}$. During the heating the toluene evaporated and the AF remained in the liqueous $5 \mathrm{CB}$ where it formed $\mathrm{J}$ aggregates at high concentrations. To obtain molecular solutions of AF toluene and chloroform were used.

To study the influence of the external electric field on the absorption spectra and photoluminescence we used the cells with transparent $\operatorname{In}_{2} \mathrm{O}_{3}$ electrodes deposited on the quartz wafers. Internal surfaces of the electrodes in the 10-micrometer thick LC cell were covered with orientant. The orientation of the nematic phase of the mixture was controlled by optical polarizing microscope. The AF dye concentration in LC was 0.5 weight $\%$ or less.

For the investigation of the photoluminescence spectra and kinetics of AF in different phases, including liquid crystalline and crystalline states of 5CB matrix, we used the technique with nanosecond resolution [8]. PL was excited by nitrogen laser $\left(\lambda=337.1 \mathrm{~nm}\right.$, pulse duration $t_{1}=8 \mathrm{~ns}$, pulse power $N=5 \mathrm{~kW}$, frequency $f=100 \mathrm{~Hz}$ ). Time-resolved PL signal was registrated by stroboscope system that provided the measurements of PL kinetics and PL spectra at different values of delay time $t_{d}$ as to maximum of the laser pulse. Time resolution of the installation for the PL lifetime measurements was about $0.1 \mathrm{~ns}$. We used SPM-2 monochromator for the spectra analysis. The spectral width of the slit varied in the limits of $0.2-0.4 \mathrm{~nm}$. The same monochromator was used for the study of absorption spectra of the solutions LC-AF.

\section{From the molecule to the crystal: photoluminescence and absorption of the solutions and crystalline films of astrofloxine}

The the formation of J-aggregates in the solutions can be proved using the PL spectra and lifetime measurements. Usually the radiation of the dyes in various solvents at low temperatures exhibits a narrow PL band with a short lifetime. In the PL spectra the band of J-aggregates is shifted to the long wavelengths as compared to the molecular solutions and the lifetime is essentially shorter. Fig. 1 shows the PL spectra of AF in different phases at $T=4.2 \mathrm{~K}$ under nitrogen laser excitation. Curves 1,2 are the PL spectra of AF solution in chloroform with the concentrations $\mathrm{C} \cong 10^{-6}$ and $10^{-3} \mathrm{~mole} / 1$, respectively. When the concentration is $\mathrm{C} \cong 10^{-6}$ mole/l the PL band at $557 \mathrm{~nm}$ with the lifetime $3.5 \mathrm{~ns}$ is observed. This spectrum corresponds to the molecular radiation of $\mathrm{AF}$. At $\mathrm{C} \cong 10^{-3}$ mole/l the $\mathrm{PL}$ spectrum shifts



Fig.1. Time-resolved photoluminescence spectra of astrofloxine solutions in chloroform (curves 1,2) and crystalline film of astrofloxine (curves 3,4 ) at $4.2 \mathrm{~K}$. 1) $t_{d}=0 \mathrm{~ns}, C=10^{-6} \mathrm{~mole} / \mathrm{l}$; 2) $t_{d}=0 \mathrm{~ns}, C=10^{-3}$ mole $/ 1$; 3) $\left.t_{d}=-3 \mathrm{~ns}, 4\right) t_{d}=15 \mathrm{~ns}$.

towards the long wavelengths by $10^{3} \mathrm{~cm}^{-1}$. In the spectrum, the bands with the maxima at 590 and $637 \mathrm{~nm}$ can be seen, which can be attributed to the formation of the aggregates of different lengths. The lifetime in the PL band at 590 $\mathrm{nm}$ is close to $1 \mathrm{~ns}$. Investigations of the PL spectra of Jaggregates with different time delays $t_{3}$ showed that these spectra are rather similar to the PL spectra of the crystalline AF films. At small values of $t_{3}$ the $590 \mathrm{~nm}$ band predominates, while with the increase of $t_{3}$ its intensity decreases as compared to the $637 \mathrm{~nm}$ band.

Curves 3 and 4 correspond to the PL spectra of the crystalline AF film measured at different time delays. At small $t_{3}$ (curve $3, t_{3}=-3 \mathrm{~ns}$ ) three bands 592,612 and $635 \mathrm{~nm}$ can be seen in the PL spectrum. The lifetime of the bands 592 and $612 \mathrm{~nm}$ is $0.4 \mathrm{~ns}$. With the increase of $t_{3}$ (curve 4 , $t_{3}=15 \mathrm{~ns}$ ) the intensity of 592 and $612 \mathrm{~nm}$ bands decreases and the band at $635 \mathrm{~nm}$ with the lifetime $2.5 \mathrm{~ns}$ predominates. One can suggest that 592 and $612 \mathrm{~nm}$ bands correspond to the radiation from the microcrystalline regions with one-dimensional ordering of the molecules while the 635 band corresponds to the radiation of the defects inside these crystalline regions. This suggestion was confirmed by the investigation of the PL of the crystalline AF films under two-photon excitation. In [9] the highly efficient twophoton transformation of IR laser radiation into visible radiation was observed at low excitation levels in several organic crystals. Further investigations demonstrated that the observed high efficiency of the two-photon absorption of the crystals under investigation is caused by high cubic nonlinearity that is peculiar for the highly-conjugated systems of $\pi$-electrons with one-dimensional ordering. Investigations of the PL with two-photon excitation at low levels of IR laser radiation can be used as a testing technique for the selection of one-dimensional highly-conjugated nanocrystalline structures with large cubic nonlinearity. 


\section{Yu. P. Piryatinski et al.: Electrooptical properties of liquid crystal ...}

PL of AF crystalline films was excited with two photons of Nd:YAGlaser radiation $\left(\lambda_{e}=1064 \mathrm{~nm}, N=0.1-0.5 \mathrm{MW}\right)$. At low excitation levels (unfocused laser beam) the spectra of the luminescence of AF films exhibit three bands with maxima at 620,650 and $700 \mathrm{~nm}$, corresponding to the crystallites of different sizes.

Investigations of the PL spectra of the AF solutions in LC 5CB demonstrated that they have the properties of both $\mathrm{J}$-aggregates in concentrated AF solutions in chloroform and J-aggregates formed in crystalline AF films. At high concentrations of AF molecules in LC the isolated J-aggregates of certain length and concentration are formed. Extra $\mathrm{AF}$ is gathers in the ordered regions of J-aggregates as colloidal particles. These colloidal particles are not uniformly distributed in the bulk of the LC but form dendrimers. With the temperature increase dendrimers are easily destroyed. Colloidal particles of J-aggregates of AF in LC 5CB can be observed in two-photon luminescence spectra at low levels of IR laser excitation. Probably, it is caused by larger length of J-aggregates in colloidal particles as compared to the one in the bulk of LC.

Fig. 2 shows the absorption spectra of AF solutions in LC 5CB for small concentrations of AF (curve 1) and for saturated solutions (curve 2). Curve 3 corresponds to the absorption of the thin crystalline film of AF. It is seen that relative intensity of the long wave length band $560 \mathrm{~nm}$ increases and somewhat shifts to longer wavelengths with the increase of AF concentration, thus this band can be ascribed to the aggregates formation. Moreover, the shoulder in the spectral range $570-600 \mathrm{~nm}$ appears in the spectrum of the concentrated AF solution. In this spectral region two bands 575 and $600 \mathrm{~nm}$ are observed in the spectra of crystalline AF films. One can suggest that both the increase of the long wavelength band and the shoulder appearance at 570-600 $\mathrm{nm}$ in absorption spectra of concentrated AF solutions in LC matrix are related to the formation of J-aggregates of different lengths. Longer J-aggregates absorb the light with the wavelengths larger than $575 \mathrm{~nm}$.

\section{Electrooptical properties of the solutions of $\mathrm{J}$-aggregates of astrofloxine in liquid crystalline matrix $5 \mathbf{C B}$}

Experimentally observed variation of the optical density of AF solution in LC 5CB under external electric field is caused mostly by LC reorientation. AF molecules, that are organized into J-aggregates and oriented along the long axes of $5 \mathrm{CB}$ molecules, follow the change of $5 \mathrm{CB}$ molecules reorientation. When the LC orientation is planar and the molecules of $5 \mathrm{CB}$ together with J-aggregates of $\mathrm{AF}$ are oriented parallel to the electrode plane, the absorption of the normal incident light in the $\mathrm{AF}$ absorption region will be the largest. The reason is that the transition dipole moment in $\mathrm{J}$-aggregate is oriented along the aggregate axis and is almost equal to zero in the perpendicular direction. Maximum absorption takes place when the E-vector is parallel to long axis of the aggregate. The nematic LC 5CB that is used as a matrix has the positive anisotropy and changes the orientation from planar to homeotropic in the electric field; thus,

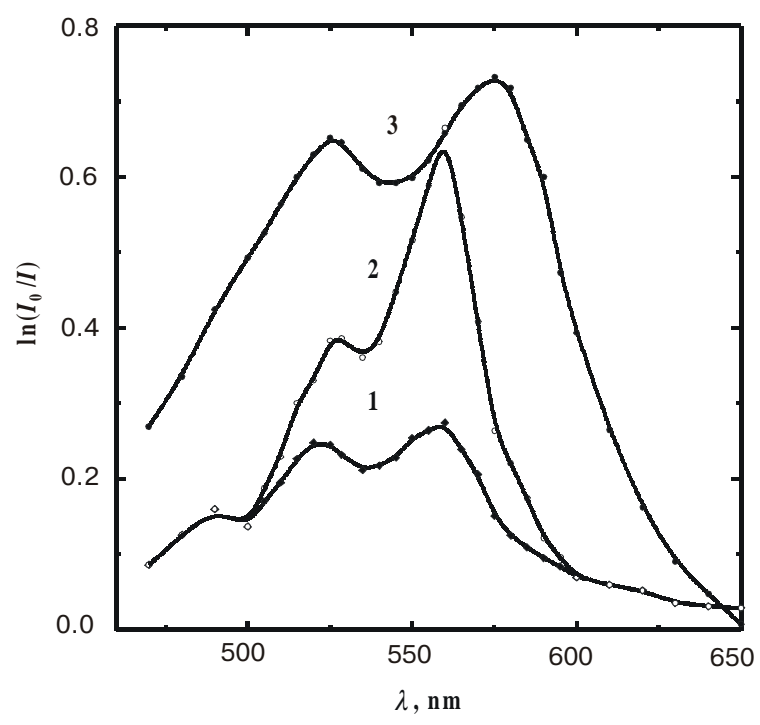

Fig.2. Absorption spectra of astrofloxine in liquid crystalline matrix of $5 \mathrm{CB}$ (curves 1,2 ) and crystalline film of astrofloxine (curve 3) at $300 \mathrm{~K}$. AF concentration is: 1) $C=0.1 \mathrm{w} . \%, 2$ ) $C=0.5 \mathrm{w} . \%$.

the dye molecules reorient perpendicular to the E-vector of the light and the sample becomes almost totally transparent. Variation of the optical density of AF solutions in LC $5 \mathrm{CB}$ in the external field in the visible range is mostly observed in the region of $\mathrm{AF}$ absorption. The reason is that 5CB LC absorbs in the UV region and at $\lambda \geq 350 \mathrm{~nm}$ the $\mathrm{LC}$ is transparent.

On the other hand, when the wavelengths of excitation, $\lambda=365$ and $337 \mathrm{~nm}$, correspond to the transparency region of $5 \mathrm{CB}$, the intense luminescence of both $\mathrm{LC}$ and dye is observed. The spectral region of LC 5CB radiation is 390$500 \mathrm{~nm}$. The intensity of PL is maximum when E-vector of incident light is parallel to the long axes of $5 \mathrm{CB}$ molecules. The PL of LC 5CB was analyzed in [8]. When LC 5CB is excited in the region of molecular absorption $(\lambda<300 \mathrm{~nm})$ monomer and excimer radiation takes place. The band of the excimer absorption is observed at $410 \mathrm{~nm}$. Taking into account that $5 \mathrm{CB}$ molecules in LC phase are not dimerized yet one may suggest that some part of the molecular pairs of $5 \mathrm{CB}$ is close to the dimer conformation and gives weak absorption at $300-400 \mathrm{~nm}$. Under the excitation of LC 5CB in this spectral range both excimer and dimer PL is observed. In the external electric field the PL anisotropy of both LC and the dissolved dye molecules changes. Therefore investigation of the PL of the solutions of AF J-aggregates in LC matrix in external fields gives additional information about their electro-optical properties.

In external field the cells with AF solution in LC 5CB exhibit pronounced electrochomic properties. Investigations of the transmission of AF solutions in LC 5CB in d.c. and a.c. electrical fields with the frequencies $1 \mathrm{~Hz}-1 \mathrm{kHz}$ and amplitude $F=1.210^{4} \mathrm{~V} \mathrm{~cm}^{-1}$ showed that the best reproducibility of the results is observed in the fields with sinusoidal modulation at the frequencies exceeding $100 \mathrm{~Hz}$. The decrease of the electric field frequency from $10^{3}$ to $10 \mathrm{~Hz}$ at 
$F=1.210^{4} \mathrm{~V} \mathrm{~cm}^{-1}$ caused the decrease of the intensity and shift of the absorption band from 564 to $556 \mathrm{~nm}$ (Fig.3). It can be ascribed to higher inertia of J-aggregates in electric field. In the d.c. electric field the AF solution in LC becomes transparent; the process of its initial properties restoration after the field switch-off is rather long. The behaviour observed can be explained by the destruction of J-aggregates in the bulk and on the electrodes surface that causes rather long restoration after field switch-off.

Electrochromic effect can be qualitatively characterized using eletrochromic coefficient $K_{e}$ that can be approximately calculated using the experimental data [11]

$$
K_{e}=\left[I(0)-(I(F) / I(0)] / F^{2} D \ln 10\right.
$$

where $D$ is the optical density of the solution at zero electric field, $I(F)$ and $I(0)$ are the intensities of the transmission light with and without field, respectively. Relative variation of light transmittance $\Delta I / I(0)=[I(0)-I(F)] / I(0)$ of the cells in the region of dye transparency at $10-10^{3} \mathrm{~Hz}$ and $F=1.210^{4} \mathrm{~V} \mathrm{~cm}^{-1}$ is shown in Fig.4. It is seen that main changes of transmission take place in the range of $\mathrm{J}$-aggregates absorption at low electric field frequencies. Depending on the electric field modulation frequency this value varies from -0.2 to -2.2

When the electric field changes from 0 to $1.2 \cdot 10^{4} \mathrm{~V} \mathrm{~cm}^{-1}$ the experimentally observed variations of the optical density of AF solution in LC 5CB are mostly caused by the reorientation of the liquid crystal that is verified by anisotropy investigations. Electrochromic effect for J-aggregates can be seen by eye. At the absence of electric field the cell is pink while in the field it becomes almost transparent.



Fig.3. Absorption spectra of astrofloxine solution in liquid crystalline $5 \mathrm{CB}$ matrix with planar orientation in a.c. electric fields at $300 \mathrm{~K}$ for $C=0.5 \mathrm{w} . \%$. 1) $\mathrm{F}=0 \mathrm{~V} \cdot \mathrm{cm}^{-1}$; 2) $F=1.2 \cdot 10^{4} \mathrm{~V} \cdot \mathrm{cm}^{-1}$; $f=100-1000 \mathrm{~Hz}$; 3) $F=1.2 \cdot 10^{4} \mathrm{~V} \cdot \mathrm{cm}^{-1}, f=10 \mathrm{~Hz}$.
Fig. 5 shows the spectra of polarized PL of the planar nematic phase of the LC with J-aggregates at $\mathrm{Hg}$ line 363 nm excitation: curve 1 - polarization of the incident light is parallel to the direction of the $5 \mathrm{CB}$ molecules and to the polarization of the measured PL signal, external electric field is absent; curve 2 - polarizations are the same as for the curve 1 but the electric field with the frequency $f=1 \mathrm{kHz}$ and amplitude $U_{0}=12 \mathrm{~V}$ is applied to the electrodes of the cell; curve 3 -incident light polarization is parallel to the direction of $5 \mathrm{CB}$ molecules and perpendicular to the polarization of the measured PL signal, external field is absent;



Fig.4. Spectral dependence of $(I(0)-I(F)) / I(0)$ for the astrofloxine solution in liquid crystalline $5 \mathrm{CB}$ matrix with planar orientation at $300 \mathrm{~K}$ for $C=0.5 \mathrm{w} . \%$. 1) $F=1.2 \cdot 10^{4} \mathrm{~V} \cdot \mathrm{cm}^{-1}$, $f=10 \mathrm{~Hz}$; 2) $F=1.2 \cdot 10^{4} \mathrm{~V} \mathrm{~cm}^{-1}, f=100-1000 \mathrm{~Hz}$.



Fig.5. Polarized photoluminescence spectra of astrofloxine J-aggregates in liquid crystalline 5CB matrix with planar orientation. $T=300 \mathrm{~K} . C=0.5$ w.\%. Other experimental conditions are described in the text. 


\section{Yu. P. Piryatinski et al.: Electrooptical properties of liquid crystal ...}

curve 4 - polarizations are the same as for the curve 3 but the electric field with the frequency $f=1 \mathrm{kHz}$ and amplitude $U_{0}=12 \mathrm{~V}$ is applied to the electrodes of the cell.

If the radiative dipole moment of the $5 \mathrm{CB}$ is parallel to the molecule axis the anisotropy of the uniaxial LC can be written as [4]:

$$
A=\left(I_{p l}-I_{p p}\right) /\left(I_{p l}+2 I_{p p}\right)
$$

where $I_{p l}$ and $I_{p p}$ are the intensities of the PL components polarized parallel and perpendicular to the polarization of the incident light, respectively.

At low AF concentrations in LC 5CB the PL spectrum of the solution (Fig. 6, curve 1) is similar to the PL spectrum of the pure LC 5CB [8]. For the planar cell the anisotropy parameter calculated in accordance with (1) is equal to 0.5 in the spectral region $390-500 \mathrm{~nm}$. In the a.c. electric field $\left(F=1.210^{4} \mathrm{~V} \mathrm{~cm}^{-1}, f=1 \mathrm{kHz}\right)$ the PL intensity decreases in the whole spectral range (curve 2) in the limits ${ }^{\Delta} I_{P L} / I_{P L}(0)=\left(I_{P L}(0)-I_{P L}(F)\right) / I_{P L}(0)=0.2-0.3$ (curve 3). Moreover the spectrum shifts to the long wavelengths by $5 \mathrm{~nm}$ which is, probably, due to the influence of the electric field on the electronic subsystem of 5CB molecules.

At high AF concentrations ( $C>0.5 \mathrm{w} . \%)$ in the $\mathrm{LC} 5 \mathrm{CB}$ solutions the PL spectra exhibit intense bands at $577 \mathrm{~nm}$ (Fig. 5, curves 1 and 2). Characteristic time and concentration dependencies of this band evidence that the long wavelengths bands in the Fig.5 correspond to the radiation of AF J-aggregates. Due to high AF aggregation degree the radiation of dye monomers is almost absent. The bands in the short wavelengths range in Fig. 5 correspond to the PL of the LC matrix molecules. Application of the electric field $\left(F=1.210^{4} \mathrm{Vcm}^{-1}, f=1 \mathrm{kHz}\right)$ to the $\mathrm{LC}$ cell with the planar

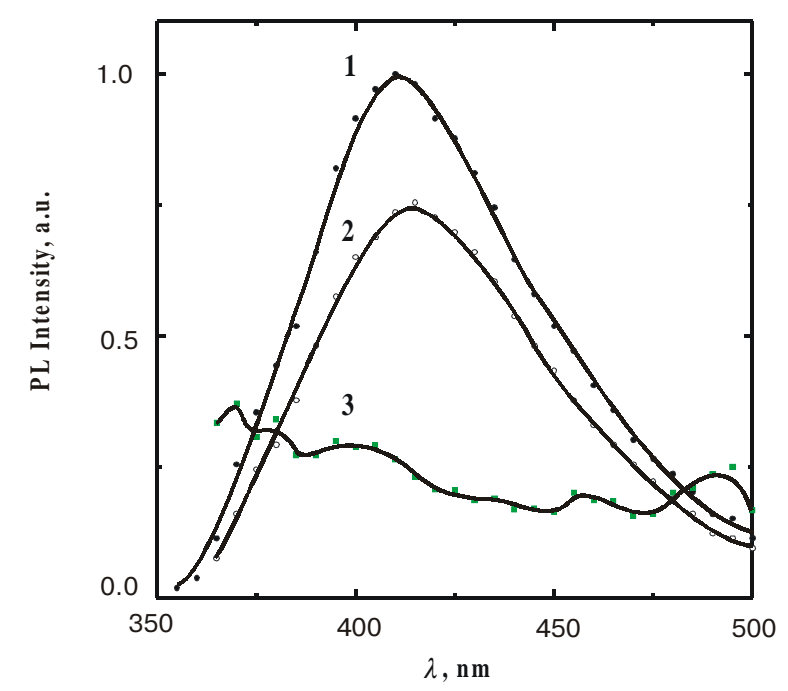

Fig.6. PL spectra (curves 1,2 ) of the astrofloxine J-aggregates in liquid crystalline $5 \mathrm{CB}$ matrix with planar orientation and $\left(\left(I_{P L}(0)-I_{P L}(F)\right) / I_{P L}(0)\right.$ (curve 3$)$ at $T=300 \mathrm{~K}$ and $C=0.1 \mathrm{w} . \%$. 1) $F=0 \mathrm{~V} \cdot \mathrm{cm}^{-1}$; 2) $F=1.2 \cdot 10^{4} \mathrm{~V} \cdot \mathrm{cm}^{-1}, f=100-1000 \mathrm{~Hz}$. orientation of the molecules causes the essential decrease of both LC and J-aggregates PL intensity. Relative change of the PL intensity in the whole spectral range $(400-600 \mathrm{~nm})$ is equal to $\Delta I_{P L} / I_{P L}(0)=0.35$.

Using (2) one can estimate (using the data in the Fig. 5) the spectral dependence of the PL anisotropy of the planar LC and J-aggregates without field (Fig.7, curve 1) and in the field (Fig.7, curve 2). At zero field the anisotropy in the PL maximum for $\mathrm{LC}$ is $A_{L C}=0.61$ and for $\mathrm{J}$-aggregates $A_{J}=0.47$. In the electric field the values of the PL anisotropy decrease. It is seen that LC matrix causes the orientation of Jaggregates parallel to the LC molecules, however, the degree of orientation order for the aggregates is lower than for 5CB molecules. Analyzing curves 2 and 4, Fig. 5 one can suppose that the obtained values of $A$ are not the saturated ones. Nevertheless, under the influence of the high frequency electric field LC efficiently reorients J-aggregates along the field. With the increase of the dye concentration in LC up to about $1 \mathrm{w} . \%$ the microcrystals (colloidal particles) of the dye «grow» in the matrix; they can be seen in the optical microscope. One can observe the reorientation of these microcrystals when the low frequency electric field is applied to the electrodes of the cell. In this case the characteristic value of the electrochromic effect is ${ }^{\Delta} I_{P L} / I_{P L}(0)=0.35$, but this is not the limiting value.

Let us analyze the case of the $\mathrm{LC}$ with the homeotropic orientation of $5 \mathrm{CB}$ molecules in the cell. For the case of ideal homeotropic orientation when 5CB molecules are strictly perpendicular to the cell surface the result is obvious. For all electric field frequencies and amplitudes and all wavelengths of the PL excitation neither 5CB not J-aggregates in LC matrix exhibit electrochromic effect. The case of the tilted orientation when LC molecules are not exactly perpendicular to the substrate reminds the case of the planar orientation. But the value $\Delta I_{P L} / I_{P L}(0)$ in this case



Fig.7. Spectral dependence of the photoluminescence anisotropy of the astrofloxine solution in the liquid crystalline $5 \mathrm{CB}$ matrix with the planar orientation at $T=300 \mathrm{~K}, C=0.5$ w. \%. 1) $F=0$ $\mathrm{V} \cdot \mathrm{cm}^{-1}$; 2) $F=1.2 \cdot 10^{4} \mathrm{~V} \cdot \mathrm{cm}^{-1}, \mathrm{f}=100-1000 \mathrm{~Hz}$. Other experimental conditions are described in the text. 
is much smaller (Fig.8). Depending on the cell (i.e. on the tilt angle of $5 \mathrm{CB}$ molecules) the value $\Delta I_{P L} / I_{P L}(0)$ can vary but it does not exceed $0.1-0.15$.

Fig.9 demonstrates the influence of the electric fields of various frequencies on J-aggregates. The field with $f>100$ $\mathrm{Hz}$ only reorients $\mathrm{J}$-aggregates along the field; the value ${ }^{\Delta} I_{P L} / I_{P L}(0)$ is constant in the whole frequency range and is close to 0.15 . Low frequency electric field with $f<10$ $\mathrm{Hz}$ efficiently destroys J-aggregates. The lower the frequency of the electric field the higher the rate of the conversion of J-aggregates into monomers. Fig. 9 shows that low frequency electric field suppresses J-band and enhances the intensity of the monomolecular PL; note, that the intensity of 5CB radiation is not essentially changed, the intensity of the aggregates PL $(\lambda>550 \mathrm{~nm})$ decreases and monomolecular radiation at $\lambda<550 \mathrm{~nm}$ increases. Destruction of aggregates is caused by electrohydrodynamic flows between the electrodes that occur in the cell in the d.c. or low frequency electric field.

\section{Influence of the electrode surface on the AF aggregation}

Investigations of the PL spectra of AF J-aggregates solutions in 5CB LC matrix showed that aggregation processes in the bulk and near the electrode are different. These differences can influence the electrooptical properties of the cell as a whole. Fig.10 shows the PL spectra of the cell with AF solution in $5 \mathrm{CB} \mathrm{LC}$ at $300 \mathrm{~K}$. The measurements were carried out for two cases of the cell filling. In the first case the cell is completely filled with LC solution of AF (curve 1). In the second case the LC with AF is pressed out of the cell (curve 2) and the layer on the electrode surface only partially fills the cell. The thickness of the subsurface layer of

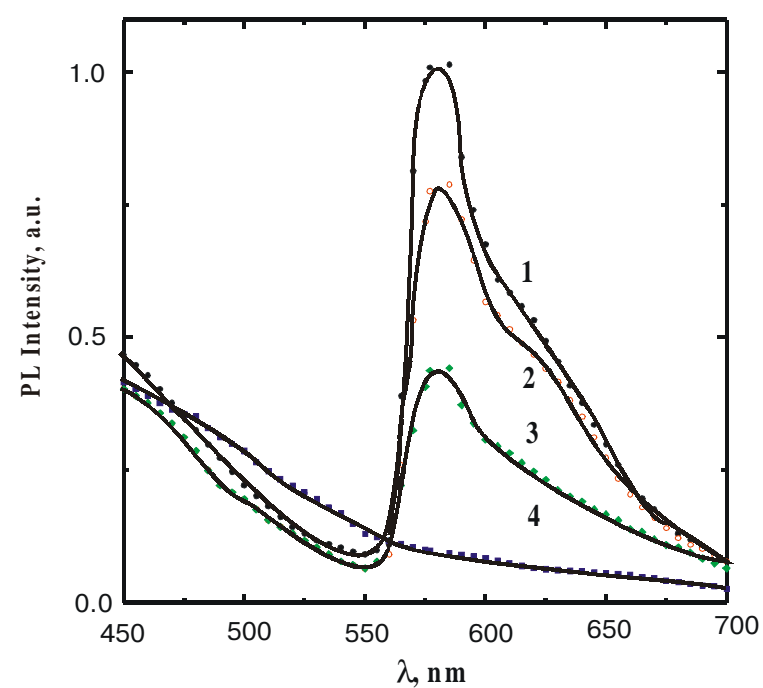

Fig.8. PL spectra of the astrofloxine J-aggregates solution in the liquid crystalline $5 \mathrm{CB}$ matrix at the tilted orientation of $5 \mathrm{CB}$ molecules: 1) electric field is absent; 2-4) the amplitude of the applied electric field is $U_{0}=12 \mathrm{~V}$ and the frequency is $100-1000 \mathrm{~Hz}$, $10 \mathrm{~Hz}$ and $1 \mathrm{~Hz}$ for 2,3 and 4 curves, respectively.
LC is smaller than the laser radiation penetration depth in LC. Relative radiation of AF molecules situated just on the surface is rather large in this case. Curve 2 in Fig. 10 is normalized using the short wavelengths part of the curve 1 . In the PL spectrum of the surface layer one can distinguish the radiation of $\mathrm{AF} \mathrm{J}$-aggregates with the maximum at 585 $\mathrm{nm}$ that is characteristic for the radiation in the bulk (curve 1). Moreover, in the differential spectrum (curve 3) which was obtained by the subtraction of the curve 1 from curve 2, two bands, 600 and $615 \mathrm{~nm}$, are seen; they can be as-

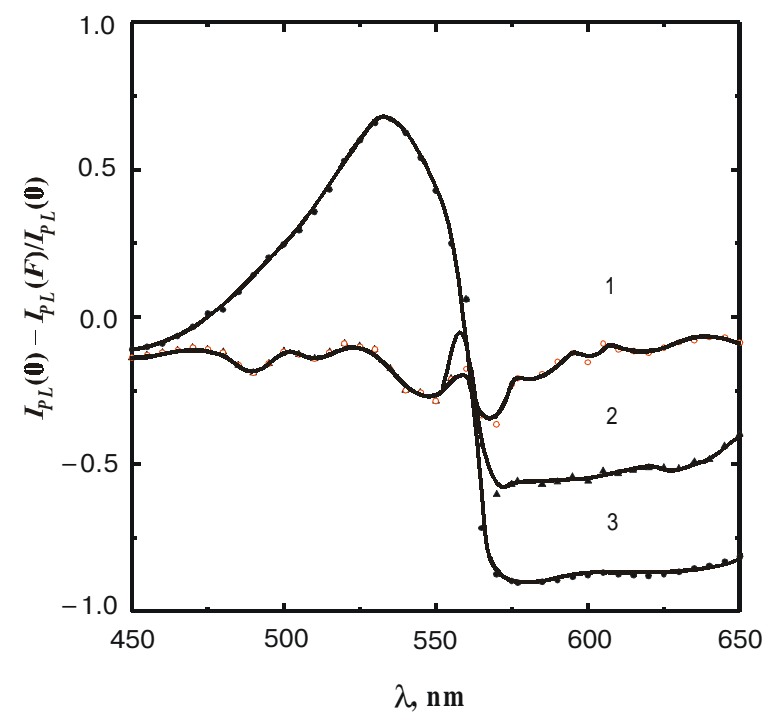

Fig.9. Spectral dependence of the $\left(\left(I_{P L}(0)-I_{P L}(F)\right) / I_{P L}(0)\right.$ for the astrofloxine $\mathrm{J}$-aggregates solution in the liquid crystalline $5 \mathrm{CB}$ matrix with planar orientation at $T=300 \mathrm{~K}$ and $C=0.5 \mathrm{w} . \%$. and $F=1.2 \cdot 10^{4} \mathrm{~V} \cdot \mathrm{cm}^{-1}$. 1) $f=100-1000 \mathrm{~Hz}$; 2) $f=10 \mathrm{~Hz}$; 3) $F-1 \mathrm{~Hz}$.

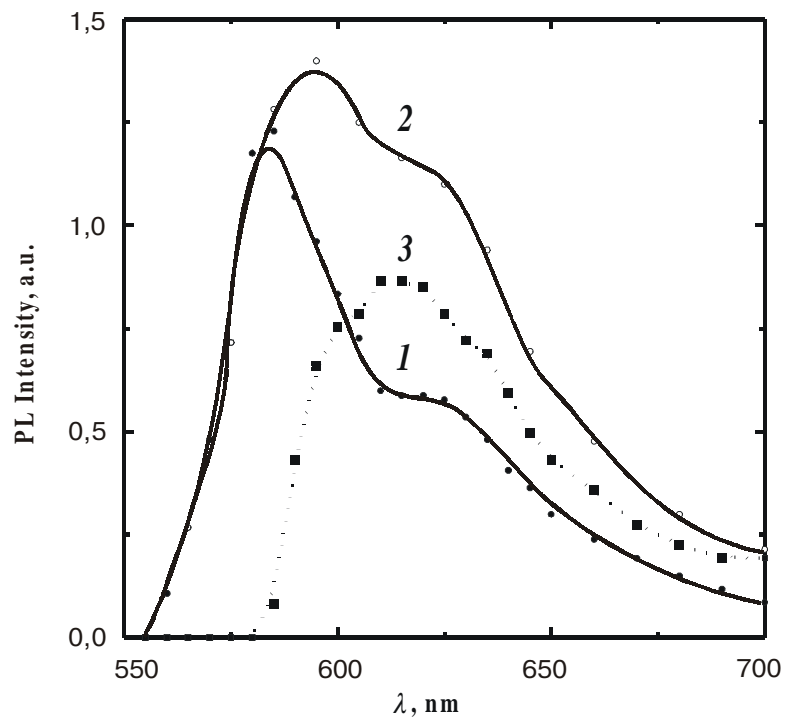

Fig.10. Photoluminescence of the astrofloxine J-aggregates solution in the liquid crystalline $5 \mathrm{CB}$ matrix at the excitation of the thick (1) and adsorbed on the electrodes surfaces (2) layers at $300 \mathrm{~K}$. 3) Differential spectrum for the curves 2 and 3. Curve 2 is normalized by the front part of the curve 1 . 


\section{Yu. P. Piryatinski et al.: Electrooptical properties of liquid crystal ...}

cribed to the formation of J-aggregates directly on the electrode surface. The formation of the surface layer of J-aggregates is influenced by various factors: Van-der-Waals and Coulomb interactions, presence of the double electric layer, temperature, solvent type, etc. The simple model of J-aggregate formation on the surface is described in [12].

Investigation of the temperature dependence of the PL anisotropy of AF solutions in 5CB LC showed that at high AF concentrations the PL anisotropy is observed even at the temperatures exceeding the temperature of LC-isotropic phase transition.

\section{Photoluminescence of the astrofloxine solution in the liquid crystalline matrix of $5 \mathrm{CB}$ at two-photon excitation}

J-aggregates are the low-dimensional organic nanocrystals and the collective interaction of the molecules in them causes their pronounced nonlinear properties. First of all the nonlinear properties of J-aggregates are caused by the high degree of $\pi$ electrons delocalization [13] that leads to anomalously high values of cubic nonlinearity. E.g., nonlinear cubic susceptibility of the solution of pseudoisocyanine J-aggregates solution was measured at room temperature by the method of four-wave light scattering and the value of $10^{-5} \mathrm{~cm}^{3} / \mathrm{erg}$ was obtained [5].

Two-photon absorption is related to the cubic susceptibility of the third order. Two-photon absorption can be described by microscopic polarization $P(\omega)$ that is proportional to the imaginary part of the cubic susceptibility $\chi^{3}$ and the square of the exciting light intensity. One can verify the above statement by calculating the generalized absorption coefficient $\alpha$ [14] that is equal to the energy absorbed in the unit volume during the unit time, averaged over the time and divided by the energy flux. If $P(\omega)=\chi^{3} E E * E(\omega) \exp [-i \omega t]$ then $\alpha=<\operatorname{Re}(J E)^{*}>/ 2 I$ where $J=\partial P / \partial t$ is the current density. Taking the differential and dividing by $I=(c / 8 \pi)|E|^{2}$ one obtains

$\alpha_{2}=\left\{32 \pi^{2} \omega / c^{2}\right\} \chi^{\prime \prime(3)}$

where $\chi^{\prime \prime(3)}$ is the imaginary part of the third order susceptibility and $\alpha_{2}$ is the coefficient in the formula $\alpha=\alpha_{0}+\alpha_{2} I^{2}$.

Our investigations showed that the AF solutions in LC exhibit intense PL under two-photon excitation, and this PL is first of all observed for colloidal particles (microcrystals). The efficiency of the IR laser radiation transformation into the visible light was as high as $10 \%$. Thus, investigations of the PL of AF J-aggregates in LC can also be used as a method of these solutions uniformity investigation. To excite the J-aggregates in colloidal particles one needs much lower intensity of the exciting light than for the excitation of isolated J-aggregates in the bulk of LC. The reason is that colloidal particles (microcrystals) consist of rather large $\mathrm{J}$ aggregates with high degree of $\pi$-electronic system conjugation. With the increase of the number of molecules in $\mathrm{J}$ aggragate the optical susceptibility of the aggregate should increase.
Fig. 11 shows the PL spectra of the AF solution in 5CB LC at $300 \mathrm{~K}$ at one- and two-photon excitation. For the twophoton and one-photon excitation the first harmonic $\left(\lambda_{e}=1060 \mathrm{~nm}, N=0.1-0.5 \mathrm{MW}\right)$ and the second harmonic ( $\lambda_{e}^{e}=532 \mathrm{~nm}, N=0.1-0.5 \mathrm{MW}$ ) of Nd:YAG laser were used, respectively. Second order dependence of the PL on the intensity of excitation was measured using the technique of the comparison with the intensity of the second harmonic of organic nonlinear crystal [9]. At the same level of excitation one- and two-phonon excited PL spectra are different. In the spectra of one-phonon excited PL two bands -595 and $633-\mathrm{nm}$ are seen; they can be ascribed to the radiation of the J-aggregates of different lengths. Apparently, in this case we observe the radiation of both isolated and incorporated into the colloidal particles J-aggregates. The spectrum of the two-phonon excited PL is shifted to the long wavelengths and is observed in the range $590-800 \mathrm{~nm}$. It consists of two bands with maxima at 650 and $700 \mathrm{~nm}$. One can suppose that the difference of the sizes of short and long $\mathrm{J}$-aggregates in solutions is essential because the radiation at $595 \mathrm{~nm}$ is noticeably less intense than in the long wave lengths range. Comparison of the intensities of the two-photon excited PL for AF J-aggregates in LC and for polydiacetelene crystals showed that in both cases it occurs at almost the same excitation levels. This evidences that the coefficients of the cubic susceptibility of these substances are comparable. For the polydiacetilene $\chi^{3}=1.4$ $10^{-11}$ CGSE units [13].

Difference of the one-and two-phonon excited PL spectra of AF J-aggregates in 5CB LC can also be caused by the influence of different states. Two-photon transitions are symmetry-forbidden in the dipole approximation for the states of different parity and are allowed for the states of the same parity. And vice versa for the one-photon transitions - the allowed are the transitions between the states of different parity.

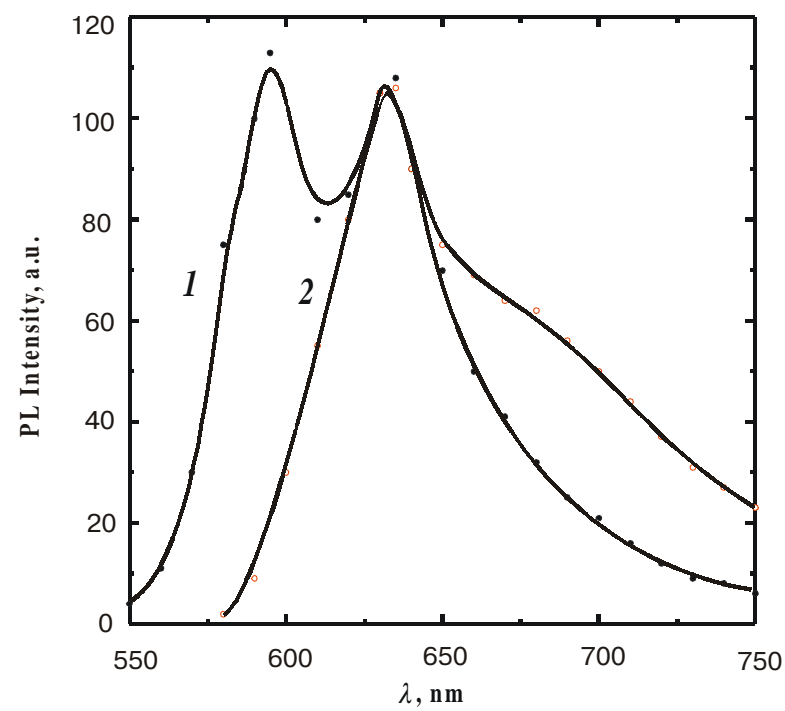

Fig.11. Photoluminescence spectra of the astrofloxine J-aggregates solution in the liquid crystalline 5CB matrix at the one-photon (curve $1, \lambda_{e}=532 \mathrm{~nm}$ ) and two-photon (curve 2, $\lambda_{e}=1064 \mathrm{~nm}$ ) excitation. 


\section{Yu. P. Piryatinski et al.: Electrooptical properties of liquid crystal ...}

\section{Conclusions}

The transmission and photoluminescence spectra of astrofloxine solutions in nematic liquid crystal are investigated in the d.c. and a.c. electric fields. AF molecules incorporated into $\mathrm{J}$-aggregates and oriented along the long axises of $5 \mathrm{CB}$ molecules follow the changes of the $5 \mathrm{CB}$ molecules orientation. When the orientation of the liquid crystal is planar and 5CB molecules with AF J-aggregates are oriented parallel to the electrode the absorption of the normal incident light in the region of the $\mathrm{AF}$ absorption has the largest value. Maximum absorption by an AF J-aggregate occurs when the E-vector of the light is parallel to the long axis of J-aggregate. In the external electric field the long axises of AF J-aggregates together with $5 \mathrm{CB}$ molecules orient perpendicular to the electrode and E-vector is perpendicular to the long axises of the J-aggregates. It this case the light absorption is minimum. Intense photoluminescence was observed under the two-photon excitation. Efficiency of the IR laser radiation transformation into the visible photoluminescence was as high as $10 \%$.

\section{References}

1. A. Adamchik, Z. Strugal'ski, Liquid crystal, «Soviet radio», pp. 60, Moscow (1989).

2. J. R. Platt, J.Chem. Phys., 34 (3) pp.862-863 (1961).

3. V. Liptej, «Modern quantum chemistry, p.274, "Mir", Moscow, 1968.

4. Yu. P. Piryatinski, V. G. Nazarenko, O. V Yatsun // Pis'ma v ZhETP 25 (19), pp.6-10 (1999).

5. V. L. Bogdanov, E. N. Viktorova, S. V. Kulya, A. S. Spiro // Pis'ma v ZhETP 53 (2) pp.100-103 (1991).

6. G. Scheibe, Optishche Anregngen organischer Systeme, p. 109, Weinheim: Verlag Chemie, 1966.

7. K. Misawa, H. Ono, K. Minoshima // Appl. Phys. Lett. 63(5), pp.577-581 (1993)

8. Yu. P. Piryatinski, O. V. Yaroshchuk // Optika i spektroskopiya 89(6), pp.942-948 (2000).

9. R. T. Deck, A. Yu. Piryatinski, Yu. P. Piryatinski, E. S. Repetski // Tech. Phys Lett. 22(1(, pp.34-38 (1996).

10. A. Piryatinski, R. Deck // Chem. Phys. Lett. 269, pp.156-160 (1997).

11. H. Labhart // Adv. Chem. Phys. 13, pp.179-204 (1967).

12. V. M. Rosenbaum, M. L. Dekhtyar' // Ukr. Fiz. Zhurn. 38(9), pp.1296-1299 (1993).

13. N. Blombergen, Nonlinear spectroscopy, p. 586, "Mir", Moscow, 1979.

14. R. Chang, T. Furtak, Surface Enhanced Raman Scattering, p. 408, "Plenum press", N.-Y. and London, 1982. 